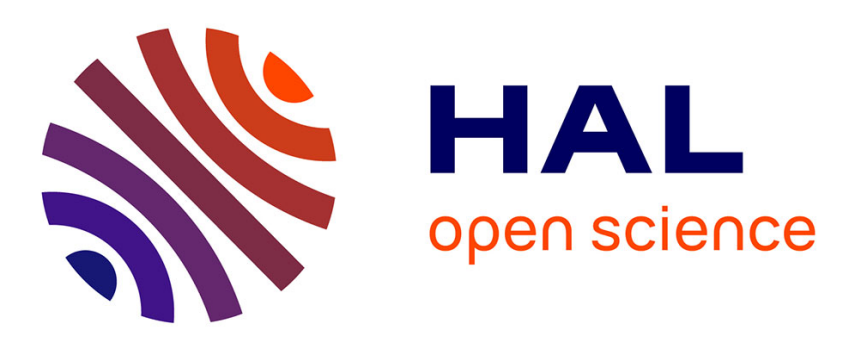

\title{
ATOMIC STRUCTURE OF AND PURE TILT GRAIN BOUNDARIES IN GERMANIUM AND SILICON
}

\author{
A. Bourret
}

\section{To cite this version:}

A. Bourret. ATOMIC STRUCTURE OF AND PURE TILT GRAIN BOUNDARIES IN GERMANIUM AND SILICON. Journal de Physique Colloques, 1985, 46 (C4), pp.C4-27-C4-38. 10.1051/jphyscol:1985402 . jpa-00224645

\section{HAL Id: jpa-00224645 https://hal.science/jpa-00224645}

Submitted on 1 Jan 1985

HAL is a multi-disciplinary open access archive for the deposit and dissemination of scientific research documents, whether they are published or not. The documents may come from teaching and research institutions in France or abroad, or from public or private research centers.
L'archive ouverte pluridisciplinaire HAL, est destinée au dépôt et à la diffusion de documents scientifiques de niveau recherche, publiés ou non, émanant des établissements d'enseignement et de recherche français ou étrangers, des laboratoires publics ou privés. 


\title{
ATOMIC STRUCTURE OF <0II> AND <00I> PURE TILT GRAIN BOUNDARIES IN GERMANIUM AND SILICON
}

\section{A. Bourret}

Centre d'Etudes Nucléaires de Grenoble, Département de Recherche Fondamentale, $85 \mathrm{X}, 38041$ Grenoble Cedex, France

Résumé - La microscopie ēlectronique haute résolution (MEHR) a été utilisée pour résoudre la structure atomique des joints de flexion $\langle 011\rangle$ et $\langle 001\rangle$ dans le germanium et le silicium. La résolution point par point actuelle donnée par cette technique, limite les renseignements sur les positions atomiques et la détermination du nombre exact d'atomes dans le plan du joint. Plusieurs unités structurales permettant de construire un joint quelconque de flexion symétrique ont été trouvées. Pour la première fois, une comparaison détaillée entre images simulées et images calculées a été effectuée dans le cas de $\Sigma=9$. Récemment la technique de MEHR a été complétée par des expériences de diffraction électronique sur le joint $\{112\} \Sigma=3$ par A.M. Papon et M.L. Petit. Ces auteurs ont proposé une structure nouvelle pour ce joint ; elle contient des liaisons reconstruites qui sont ainsi mises en évidence pour la première fois.

\begin{abstract}
High Resolution Electron Microscopy (HREM) has been used to investigate the structure of [001] and [011] pure tilt grain boundaries both nearcoincident and coincident in silicon and germanium crystals. The point-topoint resolution limit of this technique, limit the detection of the exact number of atoms and their location in the grain boundary plane. Several structural units for primary and secondary relaxations have been found. For the first time, a detailed comparison between experimental and simulated images of $\Sigma=9$ has been performed. Recently HREM has been used in conjonction with electron diffraction to solve the $\{112\} \Sigma=3$ atomic structure by Papon and Petit. These authors have proposed a new structure with reconstructed bonds.
\end{abstract}

The grain boundary (G.B) structure at the atomic level has been characterized by means of various techniques : X-ray and electron diffraction, field-ion and electron microscopy. Among these methods high resolution electron microscopy (HREM) has recently greatiy contributed to our knowledge of G.B. A relatively large number of G.B disorientations have already been observed by different authors $/ 1,2,3,4,5,6 /$. However, given the limitation of the HREM technique, this method is limited to pure tilt G.BS observed along 〈011〉 and $<001\rangle$ axes. A review of the experimental results and their interpretation will be presented after an introduction on the practical limitations given by the HREM technique. In the discussion, particular emphasis is given to the interpretation of G.B structures in terms of the structural unit model 17/. Up to now no differences have been found between germanium and silicon. Therefore general conclusions can be drawn from studies in both types of material.

I - SOLVING THE ATOMIC STRUCTURE BY HREM

\section{Resolution limit}

The contrast in a HREM image is obtained by a phase interference of diffused or diffracted beams with the central beam, resulting in an impulse response (image of one $\delta$-function) strongly depending on the defocussing distance. As a consequence the resolution limit is not uniquely defined and several distances are generally intro- 
duced : $d_{1}$ is the "Scherzer" resolution limit obtained for a special defocussing distance which gives a very simple impulse response, and $d_{2}$ is the smallest recordable distance on an image at a larger defocussing distance but with a complicated impulse response. Typical values for $d_{1}$ and $d_{2}$ are given in Table 1 .

Table I. "Scherzer" resolution limite $d_{1}$, and best resolution limit $d_{2}$ as a function of accelerating voltage. These quantities are defined for a source size equal to $0.2 \lambda^{1 / 4} C_{S}^{-1 / 4}$ and chromatic spread $0.25 C_{S}^{1 / 2} \lambda^{1 / 2}$.

\begin{tabular}{|c|c|c|c|}
\hline$E(\mathrm{keV})$ & 100 & 200 & 400 \\
\hline$C_{s}(\mathrm{~mm})$ & 0.7 & 1.05 & 1 \\
\hline$d_{1}(\AA)$ & 3.5 & 2.8 & 2.1 \\
\hline$d_{2}(\AA)$ & 2.6 & 2.1 & 1.6 \\
\hline
\end{tabular}

The number of zone axis projections which can be used as an observation direction depends critically on the "Scherzer" resolution of the instrument $18 /$. There is a considerable interest in lowering the resolution from 3 to $2 \AA$ : at $2 \AA$ level in semiconductor materials two orientations $<011\rangle$ and $<001\rangle$ can give easy lattice imaging. However the improvement down to $1.4 \AA$ which will be available very soon on some high voltage instruments will also enable to observe $\langle 111\rangle$ and $\langle 112\rangle$ zone axis. In addition, at a $1.4 \AA$ scale, all the projected atomic columns are distinguishable : this is not the case with the presently available electron microscopes where each dot in the image represents one atomic pair (in the perfect structure).

\section{Detection of the presence of any atomic column}

The first level at which a defect can be studied is to determine the number of atomic columns present in a given structure. This has important physical consequences in particular if one wants to determine the nature of the structural units present in the G.B plane. It should be emphasized that, in any case, no direct information is available along the viewing axis as an image is always two dimensional, and it will be supposed that atomic columns are complete.

In order to discuss the possibility of determining the atomistic structure it is necessary to introduce $d_{0}$, the minimum distance between projected atomic columns for the particular structure under study. Then $d_{0}$ has to be compared with $d_{1}$ and $d_{2}$ to decide whether a structure is solvable or not. In a simplified way, three cases can than be considered :

i) the minimum distance, $d_{0}$, is always larger than the "Scherzer" limit $d_{1}$. In this case all atomic columns are visible and artefacts are not introduced at Scherzer defocus : for thin specimens (typically 50-100 $\AA$ ) tunnels in the structure appears as white dots. When combined with some a priori information about atomic configuration the tunnel structure can be correlated with atomic structure.

ii) the distance $d_{0}$ is in the range between $d_{1}$ and $d_{2}$. In this case several images of a focussing series are necessary to distinguish the true structure. Artefacts such as extra spots appearing in a tunnel when atoms are bright may occur and comparison with computer simulated images is reouired. However this task is difficult and, in general, several different structures could give the same apparent final images, unless some a priori information is known.

iii) if $d_{0}<d_{2}$ the exact number of atoms included in one given spot should be determined by intensity measurement : this is particularly difficult and although theoretically possible no convincing experimental results have yet been presented in this range.

From this short and very summarized analysis the optimum conditions for observing a G.B can be predicted to be the following : 
i) d. should be as small as possible : in this respect the new $400 \mathrm{keV}$ high resolution electron microscopes should improve the results substantially (Table I).

ii) G.B observations are limited to pure tilt G.Bs along their common axis. The G.Bs are then seen end-on as only defects having a strain field independent of the $z$ axis (observation axis) can be clearly resolved : atomic columns are displaced as a whole in these conditions.

iii) specimen thickness should be as small as possible and if possible smaller than one extinction distance, typically 50-100 \&. The thickness range and the defocussing distance are two important experimental parameters which must be measured $/ 8 /$. Computer simulations including these parameters are necessary as soon as details of the order of $d_{1}$ have to be solved.

iv) the optical alignement of the microscope has to be performed carefully to ensure a good correspondance between the image and the projected structure.

\section{Location of the atomic columns}

Having determined whether or not an entire atomic column is present in the G.B plane, $i t$ is then necessary to determine the $x$ and $y$ coordinates of any projected atomic column. Matching between experimental and simulated images has to be performed using a trial and error method. It can be done visually by superposition of both images on intensity maxima. When $d_{0} \geqslant d_{1}$, this procedure gives reliable location of atomic columns whithin $\pm 0.1 d_{1}$. This sensitivity smalier than the resolution limit is due to the phase contrast which enables small phase variations of each image Fourier component to be detected. However the condition $d_{0}>d_{1}$ is generally not satisfied : in case of a $\langle 011\rangle$ direction in diamond structure one can make the same analysis on "atom-pair" column instead of single atomic column (but then the pair is supposed to be undeformed even at the G.B plane).

The rigid body translation across G.B can also be measured directly $/ 3 /$. In semiconductors this method is often sufficient to el iminate some of the proposed models (case of $\left.\{112\} \Sigma_{2}=3\right)$. However the present accuracy $\left(0.1 \mathrm{~d}_{1}\right.$ ) is not sufficiently good to measure small shift of low $\Sigma$-twin boundaries. The $\alpha$-fringes method $/ 10 /$ is much more sensitive (an order of magnitude better) in this case (for instance $\{111\}$ $\Sigma=3$ ).

\section{Chemical determination of the atomic columns}

The determination of the atom species present in the G.B plane is generally not directly possible by HREM. However HREM may sometimes complement the STEM or Auger microanatysis in an indirect way :

i) when a new phase is formed in the G.B core, impurity atoms can be recognized by the new projected atomic structure which is formed $/ 11 /$.

ii) when impurities are segregated at G.B core, they can produce large modifications in the image, specially in case of interstitial impurities or impurities with a large $Z$ difference with the matrix. However in this case, the chemical determination of the impurity is not possible, al though the segregation phenomenon can be detected by HREM.

\section{Complementary diffraction method}

In case of periodic tilt twin boundaries, the electron diffraction can give important information about the periodicities in the $z$ direction along the common axis $/ 6 /$. It is the only possible technique to detect any reconstruction along the atomic column of the common axis, and it was recently applied in addition to the HREM technique to solve the $\{112\} \Sigma=3$ structure $/ 12 /$.

\section{II - $<011\rangle$ SUB GRAIN BOUNDARIES}

Due to HREM it has been discovered that many different Burgers vectors $(\vec{b})$ exist in low angle G.Bs. Apart from the usual $60^{\circ}$ and Lomer (pure edge) $1 / 2\langle 110\rangle$ dislocation, three others $\overrightarrow{\mathrm{b}} \mathrm{s}$ have been observed : 
$b^{\prime}=\langle 111\rangle$ dissociated into three Frank partials $\left.1 / 3<111\right\rangle$

$b^{\prime \prime}=1 / 2<211>$ dissociated into three partials forming a characteristic hook-shape arrangement

$b^{\prime \prime} '=\langle 100\rangle$ non dissociated, or dissociated into two partials. Symmetric G. Bs with $\theta<10^{\circ}$ (where $\theta$, the disorientation angle, is characterized by $\theta<90^{\circ}$ for a $\{110\}$ median plane, and $\theta>90^{\circ}$ for $\{100\}$ median $\mathrm{plane}$ ), are composed of a regular array of Lomer dislocation. Symmetric G.Bs with $\theta>170^{\circ}$ are composed of a regular array of $b^{\prime \prime \prime}$ dislocation. For asymmetric configurations the exact G.B plane determines the bs or mixture of $\vec{b} s$ which are effectively present $/ 1 /$.

The structural units of the different dislocation cores are not yet completely determined. The $60^{\circ}$ dislocation along $\langle 011\rangle$, dissociated into two partials, has been studied by different authors $/ 13,14 /$ and was found to be mainly of the glide type. However the accuracy of HREM is limited and up to $50 \%$ of shuffle configuration cannot be excluded $/ 8 /$. The structural units in both cases contain dangling bonds which are supposed to be reconstructed. In the $30^{\circ}$ partial, this reconstruction should double the periodicity along the dislocation line. This is not the case in the $90^{\circ}$ partial but a shear along the dislocation appears. It should be stressed that reconstruction has not yet been observed experimentally.

The Lomer dislocation should contain a very simple structural unit here after called L-unit, with completely closed 5 and 7 atom rings. It is disappointing to realize that this configuration has not yet been observed in low angle G.B : the Lomer dislocation is always decorated by some impurities (more likely oxygen) which change completely the structural unit $/ 15 /$.

Various complicated structural units have been proposed for other dislocation types (Frank partial, stair-rods) /16/. However these dislocations act as good sinks for oxygen impurities in Ge and $\mathrm{Si}$ and they are never observed undecorated except for the $1 / 3<100>$ stair-rod, the model of which is still unclear in the central part /2/. All these hypothetical models have very disturbed bonds for which reconstruction is either possible or not : this can explain their high sensitivity to impurity decoration.

Clear and distinct similar dislocations are recognized up to $\theta \sim 10-12^{\circ}$.

\section{III - SYMMETRIC <011> TWIN BOUNDARIES}

The symmetric $<011>G$.Bs having a $\{110\}$ median plane are easy to prepare by the Czochralski method indicating that they have a low energy. Moreover in silicon polycristalline materials numerous $\langle 011>$ boundaries are present among them the $\Sigma=3$, 9 or 27 are very common. The results recently obtained by different authors are hereafter summarized for increasing disorientation angle.

$$
\text { 1. }\{255\} \Sigma=27, \theta=31^{\circ} 59 \text { (see ref. } / 5 / \text { ) }
$$

For small $\theta$ values and up to $\Sigma=27$, a symmetrical G.B is always composed of an array of L-type structural units mixed with perfect structural units of the diamond structure hereafter called $u$. At $\{255\} \Sigma=27$ this is still true but facetting starts to occur with $L$ and $L^{\prime}$ structural unit. Here' denotes the unit deduced by a mirror glide operation. However it is surprising that the sequence is $L L^{\prime} u\left(L L^{\prime}\right)$ ' and not a simple LuL' : as a consequence the projected periodicity is equal to that given by the coincidence site lattice (CSL) and not to the half periodicity of the projected CSL along $<011>$. The facetting tends to form $\{111\}$ facets along one of the crystal. The atomistic analysis of the structural units in this G.B has not been yet completely performed but the $L$ unit is the most likely.

$$
\text { 2. }\{122\}, \Sigma=9, \theta=38^{\circ} 94
$$

It is one of the most studied G.Bs /3,4/ and the only one on which complete image analysis has been performed. In the germanium case there is a rigid body translation 
$\vec{\tau}$ along $[12 \overline{2}]$ giving a small volume expansion at the interface with : $\bar{t}=(0.4 \pm 0.2) \AA$.

This result is consistent with, although greater than, the translation measured by Papon et a1. $/ 17 /$. They found $\vec{t}=0.1 \AA$ in the same direction and with the same sign using the $\alpha$-fringes method. A glide plane at the interface is compatible with 。 the symmetry of the HREM experimental images. The period along the G.B plane is $12 \mathrm{~A}$ corresponding to the periodicity of the CSL.

The Hornstra model $/ 18$ / consisting of a periodic LL'LL' ... array plus the translation $\vec{t}$, is used as a starting position to calculate simulated images (fig. 1 ).
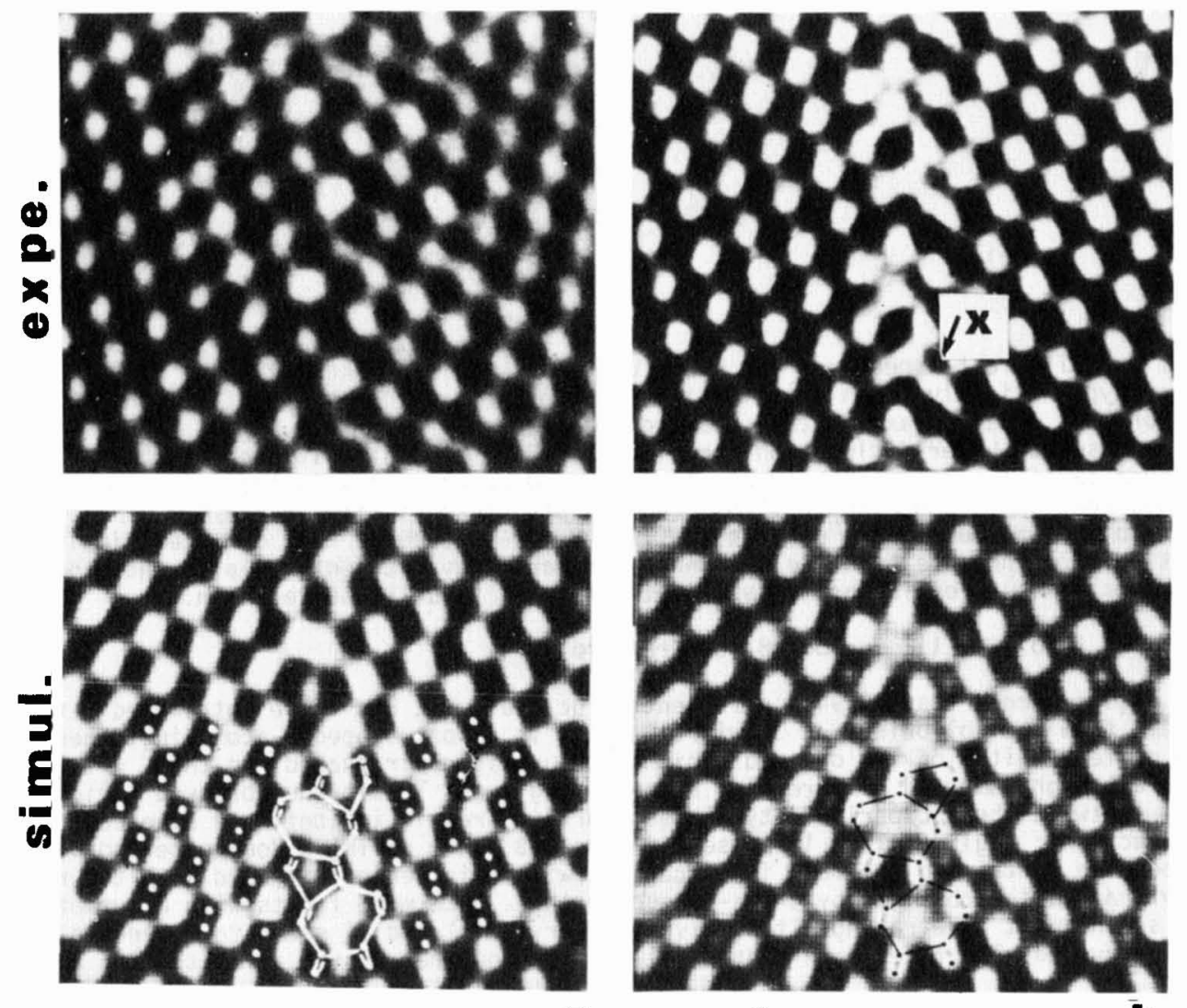

a

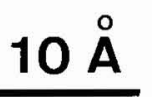

b

Fig. 1 - Twin $\{122\} \Sigma=9$ in germanium. Comparison of experimental and simulated images for two defocusing disfances a) - $500 \AA$; atoms are black, b) - $900 \AA$; atoms are white. Specimen thickness $60 \AA .200 \mathrm{keV}\left(\mathrm{d}_{1}=2.8 \AA\right)$. Differences between experimental and simulated images are particularly visible at the edge of. the 7-atom rings at $X$. Atomic location giving the best fit are represented in superposition.

Atomic columns are then moved around until the best possible fit is obtained. An excellent agreement is obtained at the "Scherzer" defocussing distance, but at the reverse contrast the fit is poor specially for the region between the 5 and 7 atomring where an elongated white dot is visible on the experimental images ( $X$ in fig. 1 ). 
When compared to the L-unit in a symmetrical Lomer dislocation (hypothetical as non experimentally observed) the unit present in $\Sigma=9$ is highly deformed and asymmetric (fig. 2). In particular the bond angles at $A$ are highly distorded ( $140^{\circ}$ instead of $110^{\circ}$ ) : it is likely that these bonds can be easily rearranged and may attract impurities. Such a segregation occuring at a slightly different site was also observed at isolated Lomer dislocation and could be attributed to oxygen impurity atoms. Similarly this segregation effect can explain the elongated dot observed close to A, however it is not possible to precise any further the location of the impurity atoms.

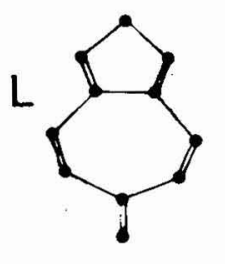

a)

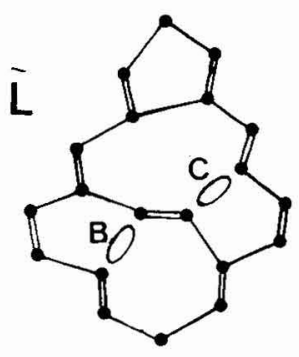

b)

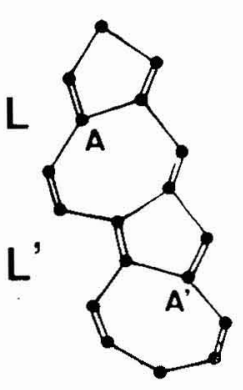

c)

Fig. 2 - Different aspects of the Lomer unit (or L-unit in the text) in

a) an hypothetically clean Lomer dislocation core (pure edge $\left.b=\frac{1}{2}<01 \overline{1}\right\rangle$ along $\langle 011\rangle$ ): Hornstra model relaxed with a Keating potential /2/

b) an observed Lomer dislocation core in a sub-G.B. Atomic positions are deduced from careful comparison between simulated and experimental images /15/. Impurity segregation is supposed to stabilize this structure at sites $B$ and $C$

c) a Lomer structural unit present in $\{122\} \Sigma=9$ as deduced from computer simulation $/ 3 /$. The site $A$ is the one where a discrepancy between simulated and experimental images remains and is attributed to some impurity segregation.

$$
\text { 3. }\{233\} \Sigma=11, \theta=50^{\circ} 48 \text { (see ref. /6/) }
$$

For angles larger than $38^{\circ} 94$ a different structural unit has to be introduced : the T-unit from the following $\Sigma=3$ twin, this unit is a boat shaped 6 -atom ring, whereas the perfect unit $u$ of the diamond cubic structure is chair shaped. In the $\Sigma=11$ case, although composed of a regular array mixing $L$ and $T$ unit, the observed periodicity is twice the CSL periodicity. This surprising result is confirmed by electron diffraction : a glide plane is present in the G.B plane giving a double periodicity. A sequence containing LL'TTL' $L$ is compatible with this periodicity and with the rigid body translation obtained by the $\alpha$-fringes method. Once more facets with a symmetrical arrangement penetrating alternatively in crystaT I and II seems to be energetically favored. The facets are along a $\{111\}$ plane of one crystal as in the $\Sigma=27$ case.

$$
\text { 4. }\{111\} \Sigma=3, \theta=70^{\circ} 53
$$

The mirror twin frequently encountered in polycristalline silicon is composed of an array of T-units. No segregation or rigid body translation has never been evidenced by HREM /3/ although a small dilatation is detectable by the more sensitive $\alpha-$ fringes techniques $/ 19 /$.

$$
\text { 5. }\{111\} \Sigma=3+1^{\circ} / 3 /
$$

This G.B close to a $\Sigma=3$ twin has additionnal periodic secondary dislocations with a $1 / 3<111>$ Burgers vector perpendicular to the G.B plane. They correspond to the insertion of a new structural unit $F$ characteristic of a Frank dislocation. A first structural unit containing 5 and 8 atoms rings with reconstruction similar to the one 
existing in the $90^{\circ}$ partial dislocation, has been proposed $/ 20 /$. However a reconstructed structure containing a supplementary atomic column pair and forming a 5-7 atoms rings is more likely and has a better correspondance to the HREM image when tunnels are imaged as white dots (fig. 3). That type of reconstruction does not affect the periodicity along the z-direction : sometimes this dislocation is dissociated emitting from the G.B plane a glissile Schokley $1 / 6<\overline{2} 1 \overline{1}>$ forming a stair-rod $1 / 6<01 \overline{1}>$ in the G.B plane. The unit is then modified in a complex manner with a different reconstruction scheme with a double periodicity along the $\langle 011\rangle$ axis $/ 20 /$.
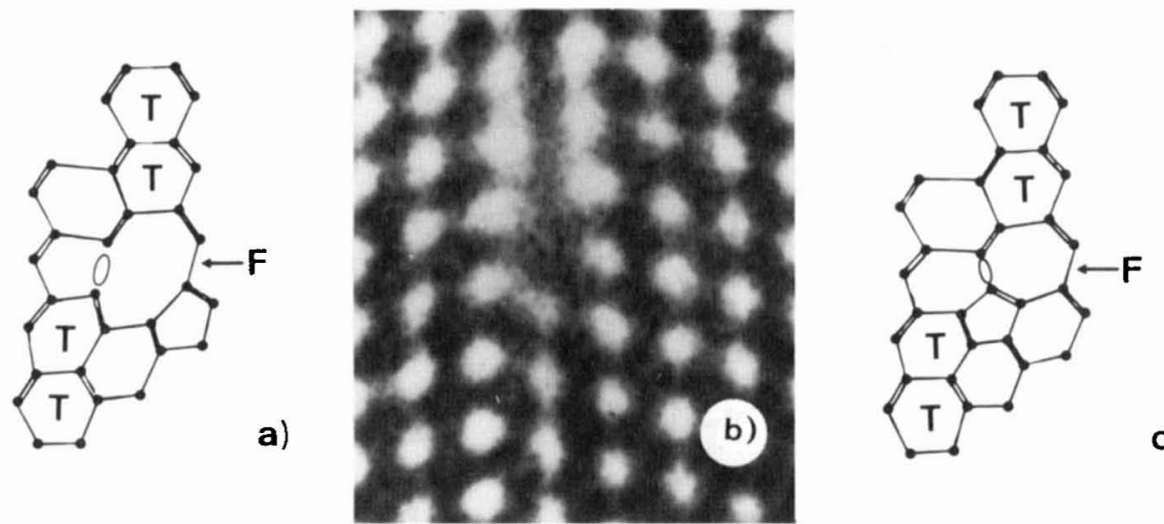

Fig. 3 - Two possible models of the F-unit $(1 / 3<111>$ defects in a $\{111\} \Sigma=3 \mathrm{G} . \mathrm{B})$ and comparison with the experimental image (200 keV, $d_{1}=2.8 \AA$, atoms are black). The model c) is more satisfactory than a) and well reproduces the special part A, $B$. Note that the type of reconstruction necessary in a) and c) is similar to the one existing in a $90^{\circ}$ partial dislocation and does not change the periodicity along the $<011>$ observation axis.

\section{6. $\{112\} \Sigma=3, \theta=109^{\circ} 47$}

This twin frequently produced in polycristalline materials has been studied by the $\alpha$-fringes method $/ 21 /$. Several models have been proposed with or without dangling bonds. However recent experimental data using combined HREM and electron diffraction have completely modified the previously proposed structural units /12/. In particular the electron diffraction pattern originating from the G.B plane (incident beam parallel to the G.B normal) exhibits superlattice spots corresponding to a double periodicity in the $z$ direction $\langle 011\rangle$, as well as in the y direction $\langle 111\rangle$ in the G.B plane (the periodicity is defined in reference to the CSL). Moreover the extinction rules show that the projected strain field along the G.B normal is centered. This double periodicity along the <111> direction is not visible by HREM when projected along the $\langle 011\rangle$ axis. A model has been recently proposed by Papon and Petit which is compatible with all these experimental results (fig. 4). It contains a new structural unit in addition to the $T$ unit, with two dangling bonds similar to those existing in the $30^{\circ}$ partial. A reconstruction of these bonds introducing a double periodicity along the <011> axis occurs as demonstrated by the diffraction pattern. Each individual pattern contains a 5-7 atom ring different from the L-unit associated with three 6-atoms rings at the reconstructed sites. This unit hereafter called $2 F$ is equivalent to a $\frac{2}{3}<111>$ dislocation. However in order to obtain the double periodicity along the $\langle 111\rangle$ direction the reconstruction occurs alternatively at two different z-level : this scheme relax partly the distortions along $\langle 011\rangle$ due to the reconstruction. The exact fit with the HREM image has not yet been performed, but this model is compatible with the periodicity observed on HREM image : the $z$ difference between two subsequent units is not visible along a <011> observation axis.

This result is very important : it is first time that a reconstruction of a $30^{\circ}$ partial type has ever been experimentally evidenced. It should be pointed out however that two facts can favor the reconstruction in the $\{112\} \Sigma=3: i)$ there are two 


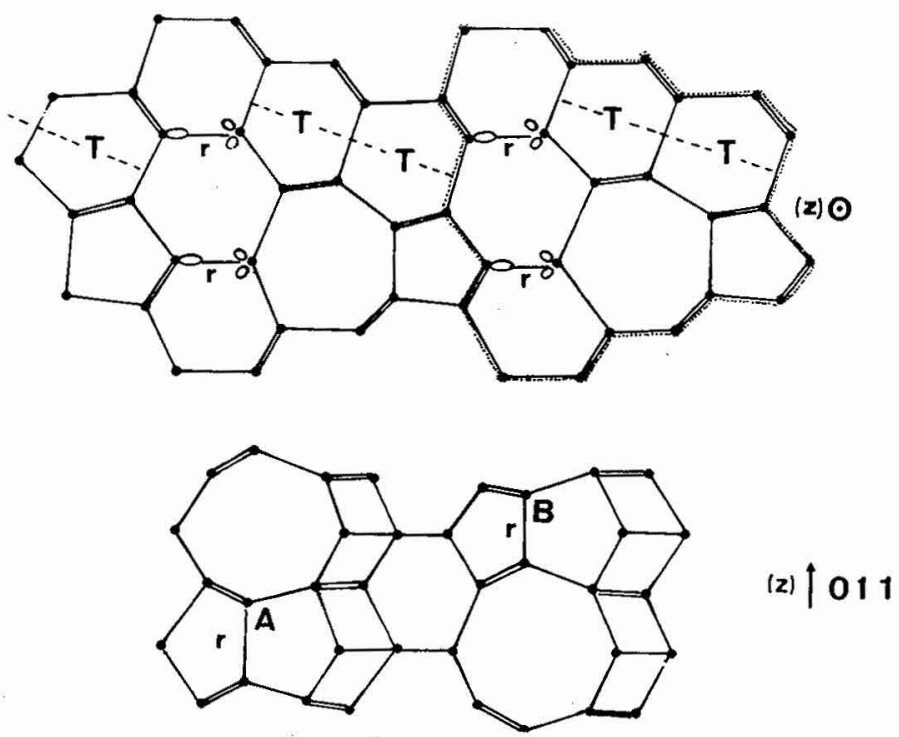

Fig. 4 - Model proposed by Papon and Petit /12/ for the $\{112\} \Sigma=3$ G.B. It consists of a succession of $2 F$-unit (outlined by a Burgers circuit) at level $z=0$ and $z=\frac{1}{2}\langle 011\rangle$ alternately along the G.B plane. Reconstruction of a pair of dangling bonds array occurs at $A$ and $B$

a) as viewed along the $\langle 011\rangle$ axis

b) viewed along the $\langle 112\rangle$ axis : the successive shift of the structure along $z$ is well visible.

close dangling bond series which can be reconstructed by inducing similar displacements along $z$. $i i)$ the alternating compressed and dilated regions introduced by a reconstruction along one series of dangling bonds are shifted by $1 / 2<011\rangle$ at the following dangling bonds series : this minimizes the strain energy at the G.B plane.

IV - SYMMETRIC <001> TWIN BOUNDARIES

Very few reliable HREM studies are available for these twins. The difficulty comes from the small distances which must be resolved $(d \sim 2 A)$. The interpretation of HREM images is very difficult as usually $d_{0}$ is in the range $d_{2} \leqslant d_{0}<d_{1}$. First qualitative observations done in germanium /3/ have shown that for $\{190\} \Sigma=41$, $\theta=12^{\circ} 68$ and $\{170\} \Sigma=25, \theta=16^{\circ} 26$ a new defect characteristic of a $\langle 100\rangle$ dislocation core appears. This core is clearly dissociated into two parts suggesting a dissociation :

$\left.\langle 010\rangle \rightarrow \frac{1}{2}\langle 011\rangle+\frac{1}{2}<01 \overline{1}\right\rangle$ with two $45^{\circ}$ dislocations; such a dissociation gives a new structural unit $/ 16 /$ which in projection shows a characteristic 3 and 5 atom-rings as suggested by Hornstra /22/.

However another possibility with two $1 / 2<110\rangle$ edge dislocations giving a different structural unit is not completely excluded. This latter case seems to be more appropriate for describing higher angle G.B such as $\Sigma=5 \%$. New instruments having point to point resolution clearly below $2 \AA$ should clarify these structures in the future.

$V-$ ASYMMETRIC $<011>$ G.BS

No systematic studies of asymmetric <011> G.B have yet been published. Nevertheless 
a few preliminary results were recently presented $/ 23 /$. An asymmetric $\Sigma=11 \mathrm{G} . \mathrm{B}$ $\left(\theta=127^{\circ}\right)$ in germanium exhibits a very complicated facetted surface. These facets occur even in the common axis direction giving a three dimensionnal microfacetting : any reliable description in terms of structural units, in this case, cannot be performed.

\section{VI - DISCUSSION}

The atomic structure of G.Bs is now commonly described in terms of a small number of structural units. This description has been extensively used by Sutton and Vitek /7/ in $\mathrm{fcc}$ metals to describe computer simulated structure. The extension to diamond cubic structures is easy and strictly equivalent to the old Hornstra model. The HREM results already obtained give support to this model but introduce some complications which were originally omitted : i) the number of units seems to be larger than previously thought, ii) the microfacetting introduces periodicities larger than the $\mathrm{CSL}, \mathrm{iii)}$ the reconstruction also introduces double periodicities compare to the CSL, iv) the impurities could modify locally the structural units.

\section{Number of structural units}

As discussed in details by Vaudin et al. $/ 5 /$ a $\langle 011\rangle$ symetric G.B up to $\theta=105^{\circ} 47$ can be constructed by mixing only few structural units. These authors limit their choice to the L-unit, the T-unit and the $2 F$-unit corresponding to the three low energy G.B, $\{122\} \Sigma=9,\{111\} \Sigma=3$ and $\{112\} \Sigma=3$. In their view any symmetrical $<011>G . B$ could be constructed by mixing the structural units of the two low energy $G$.B closest in $\theta$ value, up to $109^{\circ} 47$. The experimental results up to $\theta=70^{\circ} 53$ $\left(\Sigma=3\right.$ value) give some support to this model. However for larger angle $70^{\circ} 53$ $70^{\circ} 53<\theta<109^{\circ} 47$ this scheme is incorrect : for small angle deviation from \{111\} $\Sigma=3$, an F-structural unit is introduced periodically along the $\Sigma=3$ structure although the structure of the $\{112\} \quad \Sigma=3$ contains a completely different unit $2 \mathrm{~F}$ which cannot be analyzed as two F-units (fig. 4). This new $2 \mathrm{~F}$-unit is different from all others previously proposed. For $\dot{\theta}>109^{\circ} 47$, the experimental observations have only been performed at $170^{\circ}<\theta<180^{\circ}$ and a $<100>$ dislocation containing a new structural unit has been detected. Unfortunately the core structure has not been solved being always decorated by impurities.

In conclusion for $<011>$ symmetric G.Bs up to 5 different structural units have been observed ( $L, T, F, 2 F$ and $<100>$ ) but this number is certainly a minimum, as a large range of $\theta$ values (particularly $109^{\circ}<\theta<170^{\circ}$ ) has been completely unexplored.

For symmetrical $<001>$ G.B it is too early to make a synthesis : the experimental data are too scarce. From the first observations at $\theta>53^{\circ} 13 \Sigma=5$ two types of units have been proposed corresponding respectively to $45^{\circ}$ and $90^{\circ} 1 / 2<110>$ dislocations.

In the asymmetric <011> G.B case, the situation is hardly explored. However from the low-angle G.B case it can be inferred that a much larger number of structural units is present : $30^{\circ}$ dissociated, $90^{\circ}$ dissociated, three different Frank partials, three stair rods, in addition to the $L$ and $\langle 100\rangle$ units already mentionned.

To conclude it is necessary to point out that the structural unit model is a good way of describing the atomistic structure of $G$.BS, however the number of structural units, although limited, is certainly higher that usually thought and an atlas of this unit is far from being completed for a general G.B.

\section{Microfacetting of G.B}

Several examples of microfacetting $(\Sigma=27, \Sigma=11$, asymmetric G.B) have been observed experimentally. They generally (at least in the case of exact twin position) give a superstructure of the coincident site lattice (CSL) changing the periodicity of the G.B plane. Therefore the periodicity of the G.B plane is not uniquely descri- 
bed by the periodicity of the bicrystal. Energy minimization could induce some preferential decomposition of the G.B plane in microfacets. Up to know only double periodicity has been observed but any integer number of the CSL periodicity is possible as a new periodicity of the non planar G.B configuration. Moreover such a decomposition of a symmetric G.B in non symmetric could introduce new structural units although it has not yet been observed in simple cases.

\section{Dangling bond reconstruction}

In order to minimize the total energy, the structural units tend to have tetracoordinated silicon atoms. The $\mathrm{sp}_{3}$ hybridization is preserved even if some additional energy coming from angular misorientation or length variation of the bonds is present. For $<011>$ defects two types of reconstruction along a $<011>$ array of dangling bonds may occur in order to fulfill this requirement and restore tetracoordination. Such a reconstruction was postulated for $60^{\circ}$ dissociated dislocations and up to now was not experimentally evidenced. Therefore the result obtained by Papon and Petit is the first direct proof that reconstruction occurs and may minimize the total energy in the $\{112\} \Sigma=3$ case. As already remarked from this result it cannot be inferred that reconstruction may occur in different situations and particularly at single dislocation core. Nevertheless this reconstruction tends to stabilize a new structural unit different from the one which could have been predicted from a simple array of $1 / 3<111>$ Frank dislocation.

\section{Impurity segregation}

It is well established that impurity, specially oxygen, segregates at dislocation cores in silicon and germanium $/ 11 /$. This effect has prevented any reliable description of the "intrinsic" structural unit of individual Lomer dislocations as well as of $\vec{b}=\langle 100\rangle$ dislocations. For example the model proposed in ref. $/ 15 /$ for the Lomer dislocation core contains a modified Si atom pair (compared to the Hornstra model) the position of which is more likely stabilized by impurity segregation. Similarly in the $\{122\} \Sigma=9$ which should be composed of a series of L-unit, a careful comparison between simulated and experimental images has shown that some sites were modified by impurity segregation. This segregation is small as it does not affect the general geometry and symmetry of the G.B plane. It is however difficult to precise the type and the quantity of foreign atoms : such an uncertainty will probably remain with an improved resolution. A comparison between similar defects in different materials ( $C Z$ and $F Z$ silicon) would be very fruitful to precise this point. Interstitial oxygen atoms are attracted in highly dilatated region : they can be inserted in interstitial position between two silicon atoms either to relax strain energy or to saturate dangling bonds weakly reconstructed. On the other hand strongly reconstructed bonds (such as the one existing in $\{112\} \Sigma=3$ ) are not favorable sites for impurities and that type of structure will be rather insensitive to segregation.

\section{REFERENCES}

/1/ BOURRET A., DESSEAUX J. (1979) Phi1. Mag. A39, 405

12/ BOURRET A., DESSEAUX-THIBAULT J., LANCON F. (1983) J. de Phys. C4, 44, 15

13/ D'ANTERROCHES C., BOURRET A. (1984) Phil. Mag. A, 49, 783

14/ KRIVANEK 0.L., ISODA S., KOBAYASHI K., (1977) Phi1. Mag. 36, 331

15/ VAUDIN M.D., CUNNINGHAM B., AST D.G. (1983) Scripta Met. 17, 191

/6/ PAPON A.M., PETIT M., SILVESTRE G., BACMANN J.J. (1983) J. Microsc. Spectro. Elec. 8,135

17/ SUTTONA.P., VITEK V., (1983) Phil. Trans. Roy. Lond. A309, 1

18/ BOURRET A., THIBAULT-DESSEAUX J., D'ANTERROCHES C., PENISSON J.M., DE CRECY A., (1983) J. of Microscopy 129,337

19/ ZEMLIN F. (1979) U1tramicroscopy 4, 241

$/ 10 /$ POND R.C., VITEK V. (1977) Proc. Roy. Soc. A 357, 543

/11/ BOURRET A., COLLIEX C. (1982) Uitramicroscopy 9 , 183

112/ PAPON A.M., PETIT M.L. (1984) Scripta Met. (in press) 
113/ BOURRET A., DESSEAUX J., D'ANTERROCHES C. (1981) Inst. Phys. Conf. Ser. $\underline{60}$, The Institute of Physics p. 9

114/ ANSTIS G., HIRSCH P.B., HUMPHREYS C., HUTCHINSON J., OURMAZD A. (1981) Inst. Phys. Conf. Ser. 60, The Institute of Physics p 15

/15/ BOURRET A., DESSEAUUX J., RENAULT A. (1982) Phi 1. Mag. A45, 1

/16/ BOURRET A., D'ANTERROCHES C. (1982) J. de Phys. C1, 43, I'

117/ PAPON A.M., PETIT M., SILVESTRE G., BACMANN J.J. (1981) Materials Research Society Meeting, Boston Ed. LEAMY, PIKE, SEAGER $\underline{5}, 27$

/18/ HORNSTRA J.R. (1953) Physica 25, 409

$119 /$ POND R.C., VITEK V. (1977) Proc. Roy. Soc. London A357, 543

/20/ BOURRET A., D'ANTERROCHES C., PENISSON J.M. (1982) J. de Physique C6, 43, 83

/21/ VLACHAVAS D.S., POND R.C. (1981) Inst. Phys. Conf. Ser. 60 , the Institute of Physics p. 159

122/ HORNSTRA J. (1960) Physica 26, 198

123/ BOURRET A. (1984) J. de Physique MRS European Conf. Strasbourg (in press).

\section{DISCUSSION}

Y. Ishida: The failure of Hornstra's structure in explaining a $\Sigma=9$ boundary is certainly puzzling. Were the micrographs impossible to match by tilted images with different defocus values? Justification of your conclusion with a known structure such as a grain boundary dislocation with a screw component would be worthwhile. Was that sort of experiment performed?

A. Bourret: In fact, the Hornstra structure is the basic structure which we have observed on $\Sigma=9$. The deviation from this structure is very weak and only detectable when atomic positions are imaged as white dots; in this condition the image is much more structure sensitive. We have interpreted the small deviation from the Hornstra moder on one site as an impurity segregation. That type of segregation has been observed very clearly at Lomer type dislocations. Therefore it is not astonishing to observe similar phenomenon although on a smaller scale at $\Sigma=9$.

M. Rühle: The structure of dislocations in $\mathrm{Si}$ and $\mathrm{Ge}$ depends strongly on segregation of impurities. Will the structure of G.B. depend also strongly on impurities (present at the boundary) and what do you know about the impurities?

A. Bourret: The impurity segregation is very large at small angle grain boundaries. For large angle GB we have observed only indication of segregation for $\Sigma=9$ and even in this case it is hardly detectable. The detection limit of impurity atom by HREM in the GB plane is rather large and at least equal to a few atomic columns.

W. Gust: Do you see any chance of avoiding unwanted impurities such as oxygen and other elements influencing the grain boundary structure? 
A. Bourret: Yes, F2 silicon bicrystals should be purer. In case of polycrystalline CVD silicon it is also possible to obtain clean $G B$, however, the disorientation angle cannot be controlled as well as in a bicrystal.

D. Ast: Regarding the $\{112\} \Sigma=3$ boundary: Did you, by any chance, see different configurations? In our material (CVD) we see at least 2 and possibly 3 configurations. The boundary appears to be not very stable and generally is linear only in short sections.

A. Bourret: Papon and Petit did observe also that $\{112\} \sum=3$ was not stable and contains only short symmetrical sections. However, they investigated only the structure of the symmetrical portions in HREM.

R. Raj: Your beautiful pictures of the core structure of GBDs reflect a "tight" core structure in covalent materials. In metals, on the other hand, cores are soft and diffuse. Since grain boundary diffusivity is expected to correlate to the core structure of GBDs, this may explain why GB self diffusivities are apparently higher in metallic than in covalent crystalline materials (for example it is difficult to sinter a highly covalent material, such as silicon-nitride, without the use of dopants).

A. Bourret: In our experience the dislocation core structure in metals (titanium, molybdenum, aluminium have been already imaged) is not especially diffuse although it can be dissociated into partials as in semiconductors. Therefore, the differences in selfdiffusivity that you mention are probably due to low diffusivity of self defects in GB. A high binding energy between self interstitials or self vacancies and special sites in the GB could explain this effect. 